\title{
Metallic Pattern Prediction for Surface Wave Antennas Using Bidirectional Gated Recurrent Unit Neural Network
}

\author{
Jiashu Yang and Kin-Fai Tong \\ University College London, London WC1E 7JE, United Kingdom, e-mail: uceejy1@ucl.ac.uk; k.tong@ucl.ac.uk
}

In our recent FMCW vertical-looking radar system design [1], an antenna with cosecant-squared far-field radiation pattern is necessary for radar data processing. Conventional techniques that forms cosecant-squared pattern are incompatible with the compact radar system. Therefore, a low profile and compatible surface wave antenna, is a feasible way to achieve such radiation pattern. Curve fitting method has been performed to predict the near-zone electric field ( $E$-field) of the antenna and the predicted near-field has been utilized in the far-field transformation $[2]$.

We recently proposed a novel method to predict the metallic pattern on surface wave antennas; the desired farfield radiation pattern of the antenna is served as the input to a neural network prediction model. The method is mainly divided into two parts: i) the far-field radiation pattern to near-zone $E$-Field, and ii) from near-zone $E$-field to metallic pattern on the surface. This paper focuses on demonstrating how the metallic pattern of the antenna can be predicted by using the near-zone $E$-Field data. The near-zone $E$-field data extracted from CST simulation software is utilized as the training data for the bidirectional gated recurrent unit (Bi-GRU) neural network model. The metallic pattern attached above the substrate is converted to binary numbers as the study label of the Bi-GRU. The Bi-GRU can then be applied to predict the metallic pattern of the antenna.

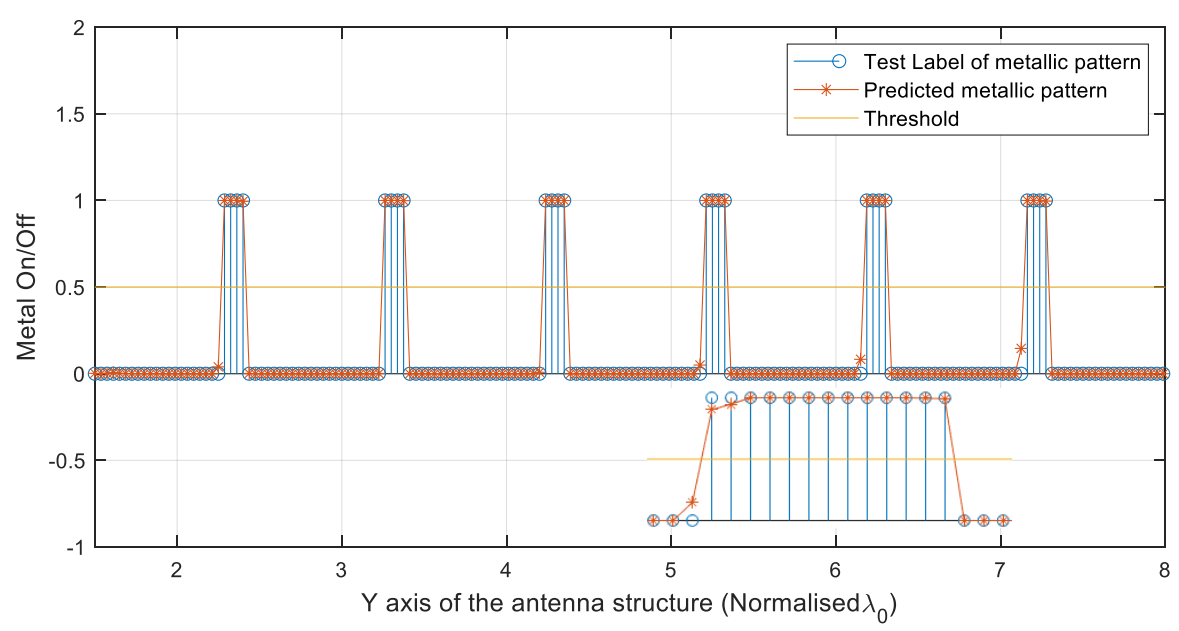

Fig. 1 Comparison between test and predicted uniformly spaced metallic patterns on the surface wave antenna

In preparing the training data stage, an array of field monitors is placed at the height of $1 \mathrm{~mm}\left(0.12 \lambda_{\mathrm{o}}\right)$ above the metallic cells along the y-direction of the proposed surface wave antenna. The magnitude of the $E_{x}$ and $E_{y}$ components of the surface wave antenna are extracted from CST simulation. The corresponding metallic pattern of the antenna model is converted to binary numbers as the training label of the Bi-GRU model. The prediction of the metallic pattern focuses on the line along the y-direction in this early stage. The line starts from the edge of the surface wave launcher and ends on the other edge of the substrate. It is divided into 800 sampling points. As shown in Fig. 1Fig. 1Error! Reference source not found, the predicted metallic pattern result of the antenna is labeled in orange asterisk. The blue circle markers are the test labels, which are the standard metallic pattern of this antenna. The $y$-axis metal on/off is the binary label of the metallic pattern where 1 indicates metal and 0 denotes no metal. The threshold will then set to be 0.5 . When the output of the Bi-GRU is less than the threshold value, it means there is no metal at such prediction point. If the output belongs to [0.5,1], it indicates there is metal. The prediction accuracy is defined as the correct predicted data points divided by the total data points. The predicted results are not necessarily the same as 0 or 1 since the predicted value has to be rounded. The metallic pattern prediction accuracy is $100 \%$ in this case.

[1] J. Yang, K. F. Tong, K. S. Lim, A. Reynolds, and C. Rawlings, "Development of Millimeter-wave FMCW Vertical-looking Entomological Radar System," 2019 IEEE Int. Work. Electromagn. Appl. Student Innov. Compet. iWEM 2019, pp. 1-2, 2019. 
[2] J. Yang and K. F. Tong, "Cosecant-Squared Radiation Pattern Surface Wave Antenna For Millimeter-wave FMCW Vertical-Looking Radar System,” 2020 IEEE Asia-Pacific Microw. Conf., pp. 801-803, 2020. 